\title{
Relationship between the 2-body Energy of the Biswas-Hamann and the Murrell-Mottram Potential Functions
}

\author{
Teik-Cheng Lim \\ Nanoscience and Nanotechnology Initiative, Faculty of Engineering, \\ National University of Singapore, 9 Engineering Drive 1, S 117576, Republic of Singapore
}

Reprint requests to Dr. T.-C. L.; Email: alan_tc_lim@yahoo.com

Z. Naturforsch. 59a, 116 - 118 (2004); received December 30, 2003

The two-body interactions in the Biswas-Hamann (BH) and Murrell-Mottram (MM) potential functions are analytically related in this paper by equating the zeroth to second differentials at equilibrium bond length. By invoking the Maclaurin series expansion for the exponential term, the MM potential function could be expressed in a manner that enables comparison of repulsive and attractive terms. Approximate and refined sets of scaling factors were obtained upon comparing the indices and coefficients, respectively. Finally, the suitability for each set of scaling functions is discussed in terms of the "softness" of the bonds.

Key words: 2-body Energy; Empirical Potential Functions; Parametric Conversion.

\section{Introduction}

Notwithstanding the lack of fundamental basis in comparison to the quantum mechanical method that requires solving the Schrödinger equation, empirical potential energy functions find their worth in computation of large-scale many-body condensed matter systems coupled with large distortion, due to their convenient execution. With various potential functions proposed, parameters in these functions were parametrized for a number of elements in crystalline, microcluster and other structural arrangements. An issue encountered for these empirical potential functions is that each of these functions requires different sets of parameters. Thus parametrization - which includes experimentation and curve-fitting - can be costly and time consuming. A more economical and rapid approach for obtaining a set of potential function parameters from another potential function is by analytical conversion of their parameters. Recently parametric relationships have been developed for converting parameters among bond-stretching potential functions [1], bond-bending potential functions [2], bond-twisting potential functions [3], and van der Waals potential functions [46]. Upon refinements [7], a prototype molecular potential function converter has been developed in a spreadsheet format [8]. However, a majority of these relationships are useful only to the chemistry community, as the parameters that were related were based on potential functions used in physical/computational chemistry softwares. Within the context of condensed matter, Stoneham et al. [9] performed a comparison of eight valence-force potentials for silicon. However, valence-force potentials are useful only for describing small distortions from equilibrium. A group of six potential functions was compared in detail by Balamane et al. [10], but these functions were studied without analytical conversion amongst the potential functions. In this paper, a set of analytical relationships is developed for converting parameters among the 2-body interactions of the Biswas-Hamann (BH) and the MurrellMottram (MM) potential functions. $\mathrm{BH}$ and $\mathrm{MM}$ for parametric connection was selected because these two potential functions were developed for silicon [1113]. Another reason for their selection was that both $\mathrm{BH}$ and $\mathrm{MM}$ belong to a class of potential functions where the total energy of a system is the sum of the energy in the 2-body and 3-body interactions:

$$
\Phi=\phi_{2-\text { body }}+\phi_{3-\text { body }}=\sum_{i<j} U_{i j}+\sum_{i<j<k} W_{i j k} .
$$

Generally speaking, (1) is a subset of the BornOppenheimer approximation. However higher moments (e.g. 4-body and higher) are neglected due to quick convergence [14]. Specifically, the 2-body interactions for the $\mathrm{BH}$ and $\mathrm{MM}$ potential functions were given as

$$
U_{\mathrm{BH}}=A_{1} \exp \left(-\lambda_{1} r\right)+A_{2} \exp \left(-\lambda_{2} r\right)
$$




\begin{tabular}{lll}
\hline Potential functions and parameters & Parametric relationships & Scaling factors \\
\hline & $A_{1}=D\left(\frac{\xi_{2}}{\xi_{1}-\xi_{2}}\right) \exp \left(\xi_{1}\right)$ & Approximate: \\
$U_{\mathrm{MM}}=U_{\mathrm{MM}}(D, a, R, r)$ & $A_{2}=D\left(\frac{\xi_{1}}{\xi_{1}-\xi_{2}}\right) \exp \left(\xi_{2}\right)$ & $\left\{\begin{array}{l}\xi_{1} \\
\xi_{2}\end{array}\right\}=a\left\{\begin{array}{l}2 \\
1\end{array}\right\}$ \\
$U_{\mathrm{BH}}=U_{\mathrm{BH}}\left(A_{1}, A_{2}, \lambda_{1}, \lambda_{2}, r\right)$ & $\lambda_{1}=\frac{\xi_{1}}{R}$ & Improved: \\
& $\lambda_{2}=\frac{\xi_{2}}{R}$ & $\left\{\begin{array}{l}\xi_{1} \\
\xi_{2}\end{array}\right\}=a\left\{\begin{array}{l}\sqrt{2} \\
(1 / \sqrt{2})\end{array}\right\}$ \\
\hline
\end{tabular}

Table 1. Parametric relationships and scaling factors between BH and MM 2-body potentials. and

$$
U_{\mathrm{MM}}=-D\left[1+a\left(\frac{r}{R}-1\right)\right] \exp \left(-a\left(\frac{r}{R}-1\right)\right),
$$

respectively. The relationship between $\mathrm{BH}$ parameters $\left(A_{i}, \lambda_{i}\right.$ for $\left.i=1,2\right)$ and MM parameters $(D, a, R)$ is developed in the next section.

\section{Analysis}

Equating the 2-body $\mathrm{BH}$ and $\mathrm{MM}$ potential functions from the zeroth to the second differential with respect to the interatomic distance, $r$, at the equilibrium bond length, $R$,

$$
\left(\frac{\partial^{n} U_{\mathrm{BH}}}{\partial r^{n}}\right)_{r=R}=\left(\frac{\partial^{n} U_{\mathrm{MM}}}{\partial r^{n}}\right)_{r=R} \quad ; n=0,1,2,
$$

we have the following 3 independent equations:

$$
\begin{aligned}
& A_{1} \exp \left(-\xi_{1}\right)+A_{2} \exp \left(-\xi_{2}\right)=-D, \\
& \xi_{1} A_{1} \exp \left(-\xi_{1}\right)+\xi_{2} A_{2} \exp \left(-\xi_{2}\right)=0, \\
& \xi_{1}^{2} A_{1} \exp \left(-\xi_{1}\right)+\xi_{2}^{2} A_{2} \exp \left(-\xi_{2}\right)=a^{2} D,
\end{aligned}
$$

with scaling factors

$$
\xi_{i}=\lambda_{i} R \quad ; \quad(i=1,2) .
$$

Eliminating the terms $A_{i} \exp \left(-\xi_{i}\right)$ for $i=1,2$ from (5) - (7) and solving simultaneously leads to the upper and lower solutions

$$
\left\{\begin{array}{l}
\xi_{1}^{\text {upp }} \\
\xi_{1}^{\text {low }}
\end{array}\right\}=\left\{\begin{array}{c}
\xi_{2} \\
\left(a^{2} / \xi_{2}\right)
\end{array}\right\} .
$$

By applying (6) and (8), the original 2-body $\mathrm{BH}$ potential given by (2) can be expressed as

$$
\begin{aligned}
\frac{U_{\mathrm{BH}}}{A_{1}}= & \exp \left(-\xi_{1} \frac{r}{R}\right) \\
& -\frac{\xi_{1}}{\xi_{2}} \exp \left[-\xi_{1}+\xi_{2}\left(1-\frac{r}{R}\right)\right] .
\end{aligned}
$$

Eliminating the term $A_{2} \exp \left(-\xi_{2}\right)$ from (5) and (6) gives

$$
A_{1}=D\left(\frac{\xi_{2}}{\xi_{1}-\xi_{2}}\right) \exp \left(\xi_{1}\right)
$$

which, upon substitution into (10), leads to the loose form of (2)

$$
\begin{aligned}
\frac{U_{\mathrm{BH}}}{D}= & \left(\frac{\xi_{2}}{\xi_{1}-\xi_{2}}\right) \exp \left[\xi_{1}\left(1-\frac{r}{R}\right)\right] \\
& -\left(\frac{\xi_{1}}{\xi_{1}-\xi_{2}}\right) \exp \left[\xi_{2}\left(1-\frac{r}{R}\right)\right] .
\end{aligned}
$$

As such, (12) shows that the upper solution of (9) is invalid. In paving a way for relating parameters of these potential functions, we rewrite the MM potential in (3) as

$$
\frac{U_{\mathrm{MM}}}{D}=\left[a\left(1-\frac{r}{R}\right)-1\right] \exp \left[a\left(1-\frac{r}{R}\right)\right] .
$$

Upon recalling the Maclaurin series expansion of the exponential term

$$
\exp \left[a\left(1-\frac{r}{R}\right)\right]=\sum_{n=0}^{+\infty} \frac{a^{n}(1-r / R)^{n}}{n !},
$$

we have the approximation

$$
a\left(1-\frac{r}{R}\right) \approx \exp \left[a\left(1-\frac{r}{R}\right)\right]-1
$$

which, upon substitution into (3), gives the approximate $\mathrm{MM}$ function

$\frac{U_{\mathrm{MM}}^{\mathrm{approx}}}{D}=\exp \left[2 a\left(1-\frac{r}{R}\right)\right]-2 \exp \left[a\left(1-\frac{r}{R}\right)\right]$.

Now, comparison of the exponential indices of (12) and (16) gives $\xi_{1}=2 a$ and $\xi_{2}=a$. On the other hand, comparing the coefficients to the exponential terms these equations generally indicate that

$$
\xi_{1}=2 \xi_{2} \text {. }
$$




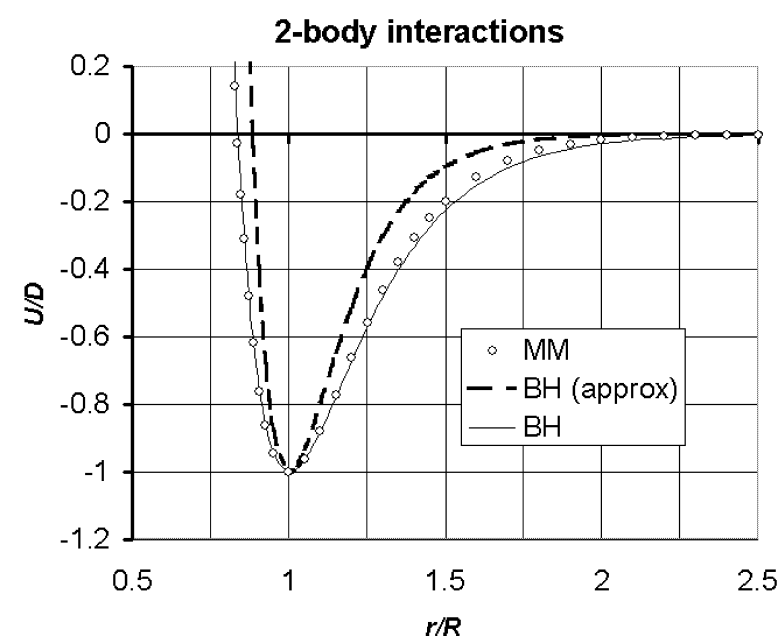

Fig. 1. Plots of $\mathrm{BH}$ curves based on conversion from $\mathrm{MM}$ parameters.

Hence substitution of (17) into the lower solution of (9) leads to $\xi_{1}=\sqrt{2} a$ and $\xi_{2}=a / \sqrt{2}$. A summary of the parametric relationships for the 2-body interactions of the $\mathrm{BH}$ and $\mathrm{MM}$ potential functions is furnished in Table 1.

\section{Results and Discussion}

For verification of the developed parametric relationships, curves of non-dimensional 2-body $\mathrm{BH}$ energy were plotted against the non-dimensional interatomic distance based on MM parameters. Parametrized values of $a$ are 5.5 for silicon crystals $[12,13]$, and 6.5 for silicon microclusters [15]. We select the average value of $a=6$ for convenient illustration. Figure 1 shows a plot of the 2-body MM curve, and the corresponding $\mathrm{BH}$ curves based on parametric conversion from $\mathrm{MM}$ parameters. We observe an

[1] T. C. Lim, J. Math. Chem. 33, 29 (2003).

[2] T. C. Lim, J. Math. Chem. 32, 249 (2002).

[3] T. C. Lim, J. Math. Chem. 31, 421 (2002).

[4] T. C. Lim, J. Math. Chem. 33, 279 (2003).

[5] T. C. Lim, J. Math. Chem. 34, 221 (2003).

[6] T. C. Lim, Z. Naturforsch. 58a, 615 (2003).

[7] T. C. Lim, MATCH Commun. Math. Comput. Chem. 49, 155 (2003).

[8] T. C. Lim, MATCH Commun. Math. Comput. Chem. 50, 185 (2004).

[9] A. M. Stoneham, V. T. B. Torres, P. M. Masri, and H. R. Schober, Phil Mag. A 58, 93 (1988). overestimation when the BH curve is plotted based on the approximate conversion from MM parameters. When the refined relationship is used, the $\mathrm{BH}$ curve gives an excellent correlation to the MM curve. The higher slope at the inflexion point corresponding to the approximate $\mathrm{BH}$ curve suggests its usefulness in curve-fitting "hard" bonds, which exhibit a steep rise in energy from equilibrium, followed by an abrupt leveling off of the 2-body interaction energy. The lower slope at the inflexion point corresponding to the refined BH curve indicates its usefulness in curve-fitting of "soft" bonds, characterized by a more gradual rise in 2-body energy from the equilibrium point. Discrepancies may well be attributed to the approximation adopted in (15) but, as evident from Fig. 1, the discrepancy is insignificant when the refined relation is used.

\section{Conclusions and Suggestion}

A method for theoretically relating the 2-body potential energy functions in the $\mathrm{BH}$ and $\mathrm{MM}$ potentials has been described and graphically verified. Essentially, both potential functions were equated at the equilibrium bond length for the zeroth, first and second differentials. Comparison of the repulsive and attractive terms gives two sets of scaling factors. An approximate and a refined set of scaling factors is obtained when the indices and the coefficients are equated, respectively. The approximate relations give a higher estimation of the BH curve based on the MM parameters - thereby more suited for curve-fitting "hard" bonds, while the refined relation gives excellent correlation. It is suggested that 3-body potentials in the $\mathrm{BH}$ and $\mathrm{MM}$ potential functions are considered in future work.

[10] H. Balamane, T. Halicioglu, and W. A. Tiller, Phys. Rev. B 46, 2250 (1992).

[11] R. Biswas and D. R. Hamann, Phys. Rev. Lett. 55, 2001 (1985).

[12] J. N. Murrell and R.E. Mottram, Mol. Phys. 69, 571 (1990).

[13] J. N. Murrell and J. A. Rodriguez-Ruiz, Mol. Phys. 71, 823 (1990).

[14] J. N. Murrell, S. Carter, S. C. Farantos, P. Huxley, and A. J.C. Varandas, Molecular Potential Energy Functions, Wiley, New York 1984.

[15] S. Li, R.L. Johnston, and J. N. Murrell, Chem. Soc. Faraday Trans. 88, 1229 (1992). 